\title{
La constitution d'une terminologie de l'éloquence française à partir des nomenclatures rhétoriques du grec et du latin
}

Constitution of a Terminology of French Eloquence Based on Greek and Latin

Rhetorical Nomenclatures

\section{Christian Nicolas}

\section{(2) OpenEdition}

\section{Journals}

Édition électronique

URL : https://journals.openedition.org/recherchestravaux/3915

DOI : 10.4000/recherchestravaux.3915

ISSN : 1969-6434

Éditeur

UGA Éditions/Université Grenoble Alpes

\section{Édition imprimée}

ISBN : 978-2-37747-326-7

ISSN : 0151-1874

\section{Référence électronique}

Christian Nicolas, « La constitution d'une terminologie de l'éloquence française à partir des nomenclatures rhétoriques du grec et du latin », Recherches \& Travaux [En ligne], 99| 2021, mis en ligne le 08 décembre 2021, consulté le 11 décembre 2021. URL : http://journals.openedition.org/ recherchestravaux/3915; DOI : https://doi.org/10.4000/recherchestravaux.3915

Ce document a été généré automatiquement le 11 décembre 2021.

(c) Recherches \& Travaux 


\title{
La constitution d'une terminologie de l'éloquence française à partir des nomenclatures rhétoriques du grec et du latin
}

\author{
Constitution of a Terminology of French Eloquence Based on Greek and Latin \\ Rhetorical Nomenclatures
}

Christian Nicolas

1 L'éloquence est la mise en pratique d'une théorie dotée d'une méthodologie et d'un lexique technique de spécialité. Mais le cheminement s'est opéré sans doute en sens inverse, comme dans toute discipline. En effet, la constitution d'une pratique sociale en sciences passe par des phases intellectuelles diverses qui, pour le dire vite, tendent à ériger un savoir-faire en savoir : définition du domaine concerné ; mise en place d'une méthodologie; mise en place d'une théorie, laquelle s'appuie sur une terminologie. Alors peut se déployer à nouveau la pratique, cette fois étayée par des connaissances livresques qui se transmettent.

Dans le cas de la rhétorique, c'est une activité qui se déploie d'abord, au moins pour ce qui est du monde occidental, dans le cadre d'une pragmatique judiciaire, dans un espace-temps précis (la Sicile des tyrans du $v^{e}$ siècle avant notre ère); puis elle est l'objet de préceptes écrits destinés à acquérir une technique de défense par la persuasion : deux recueils, perdus pour nous, furent écrits par les philosophes Corax et Tisias, attaqués par Gorgias de Léontium, dans le cadre spatio-temporel précis des procès de Sicile par lesquels des propriétaires expropriés par les tyrans essayaient de recouvrer leurs biens ${ }^{1}$. Mais c'est évidemment Aristote qui, au IV siècle, est le premier théoricien de ce nouvel art, cette fois abstrait du contexte judiciaire sicilien qui l'avait fait naître et généralisé à la parole dans toute sa portée, y compris celle de l'écrit.

Bien sûr, on n'a pas attendu Aristote pour faire de la rhétorique : Ulysse et Nestor sont de grands orateurs de l'épopée homérique, dont on admire la force de persuasion. Mais 
on est là à l'époque du savoir-faire et c'est Aristote qui fonde l'époque du savoir technique.

Dans le tout début de sa Rhétorique, Aristote, non sans polémique, explique pourquoi les ouvrages qui ont précédé le sien ne sont pas des traités techniques stricto sensu : à ces prédécesseurs (sont dans son collimateur implicitement Corax, Callippe, Pamphile,

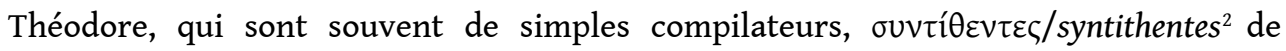
recettes), il a manqué « de se définir à eux-mêmes leur fonction ("̌pyov/ergon), le sujet propre et distinct de leur art $(\pi \rho \tilde{\alpha} \gamma \mu \alpha / p r a ̂ g m a)$, d'en mesurer toute l'étendue, d'en discerner toutes les parties ${ }^{3}$. Autant de points qui structurent le traité aristotélicien et en font le fondement de la discipline érigée en savoir.

La Rhétorique d'Aristote, en tant que texte fondateur, a été abondamment lue et commentée. Elle a aussi été adaptée en d'autres langues, en latin d'abord, dans les langues modernes ensuite. Et ce que nous voudrions montrer ici, c'est que la migration d'une terminologie technique d'une langue à une autre n'est que rarement exempte d'accidents, parfois bénéfiques.

6 La première migration importante se fait naturellement du grec vers le latin. Et le latin a recours à trois procédés principaux pour opérer ce transfert.

\section{L'emprunt lexical}

7 Il est rare qu'un terme technique grec n'ait pas au moins une occurrence en latin, en tant qu'il est le point de départ du processus de naturalisation. On cite le terme, parfois sa source (Aristote, Hermogène, Platon, etc.), en l'état : c'est l'emprunt lexical.

On peut supposer que l'acclimatation du terme est progressive et que le cheminement de cet emprunt lexical mène du xénisme, écrit et décliné en grec, à l'intégration pleine et entière du mot, translitéré en alphabet latin et décliné à la latine, via le pérégrinisme. Le processus s'accompagne généralement d'un appareillage métalinguistique fort qui s'épure progressivement jusqu'à disparaître complètement, quand le mot étranger est si bien intégré qu'on n'a plus besoin d'insister sur sa forme. Le parcours standard du terme candidat à la naturalisation est le suivant :

- Phase 1, dite phase métalinguistique autonymique, dans laquelle le terme grec représente le nom de la chose. Par exemple, Cic. Ac. 2.136: "[...] mirabilia Stoicorum, quae $\pi \alpha \rho \alpha ́ \delta \circ \xi \alpha$ nominantur » («[...] les énoncés surprenants des Stoïciens, qui s'appellent paradoxa»): on voit que le terme grec autonyme, sous sa forme graphique grecque, ne désigne pas la chose mais le mot.

- Phase 2, dite phase à connotation métalinguistique autonymique, elle-même à étapes, lors de laquelle le terme passe de la désignation de la chose dénommée à celle de la chose

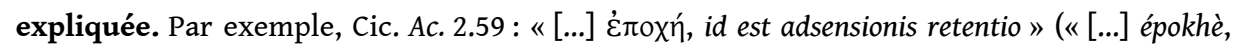
c'est-à-dire "suspension de l'assentiment" ») : le mot désigne (presque) la chose mais a encore besoin d'un commentaire métalinguistique.

- Phase 3, dite phase de l'aboutissement de l'emprunt, où le terme allogène est pleinement considéré comme autochtone. Par exemple, Cic. Or., 114: «[...] atque etiam ante hunc Aristoteles principio Artis rhetoricae dicit illam artem quasi ex altera parte respondere dialecticae » («[...] et même, avant lui, Aristote au début de sa Rhétorique dit que cet art correspond pour sa deuxième partie à la dialectique ») : les termes rhetoricae ou dialecticae désignent ici des 
choses (non des mots) et sont intégrés à l'énoncé latin en qualité de génitifs de première déclinaison ${ }^{4}$.

\section{Le calque morphologique}

9 Les deux langues antiques sont typologiquement si proches que le traducteur ou l'adaptateur de terminologie a des réflexes difficiles à contrer. Le technicisme à adapter est presque toujours un mot formé (dérivé ou composé), qui a une certaine transparence sémantique dans la langue-source. Dès lors, au lieu de l'emprunter avec sa forme brute et inanalysable pour un lecteur non hellénophone, on peut choisir de le traduire morceau par morceau, en profitant de ce que l'identité structurelle entre les deux langues permet de le faire dans des conditions optimales, puisque mots grecs et latins se forment de la même façon, avec la même syntaxe interne (préfixe, radical, suffixe, désinence casuelle, par exemple). Ce procédé de traduction de la forme interne est ce que l'on appelle le calque morphologique.

Par exemple, Quintilien (Quint. 1.5.22), évoquant la question de l'accentuation, écrit :

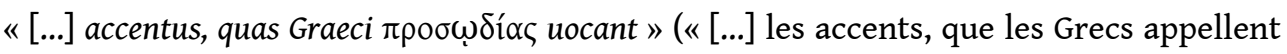
du nom de prosôdia»). Malgré les apparences, il ne se contente pas de signaler incidemment ou pour faire de l'encyclopédisme que les Latins disent accentus et les Grecs prosôdia pour désigner l'accent. Il fait implicitement une équivalence morphologique, car le néologisme choisi (qui n'est pas le mot tonus ou tenor, par exemple) est le décalque strict de son modèle grec : ad-est la traduction automatique de $\pi \rho \circ \sigma-($ pros-), -cen-, avatar de cano « chanter ", est la traduction automatique de - $\omega \delta$ $(-\hat{o}<i>d-)$ qui, comme son avatar verbal ợ $\delta \omega(-\hat{a}<i>d-)$, renvoie à la notion de «chant ", et le tout se termine dans chaque langue par un suffixe de nom d'agent. Pour nous, francophones, qui connaissons familièrement le mot français accent, le mot latin accentus est transparent; mais au moment de son apparition en latin, créé pour correspondre à son modèle $\pi \rho \circ \sigma \omega \delta$ ía avec le sens parallèle de « à/en + chant + suffixe d'action", il devait créer la même surprise que chez nous si nous tentions de «traduire » ac-cen-tus par des morphèmes français. En français, traduire accentus par accent c'est, ni plus ni moins (et sans même s'en rendre compte), faire un emprunt lexical (savant) au latin, un emprunt complètement intégré dès son arrivée ; mais la traduction stricto sensu par calque morphologique donnerait peut-être «en-chantement $»$.

11 Parfois, faute de parvenir à une solution univerbée, le latin technique propose une lexie. Mais on constate que le plus souvent l'adaptateur met les mots de la lexie dans l'ordre qui correspond à celui des morphèmes du grec, comme dans ce passage de Quintilien :

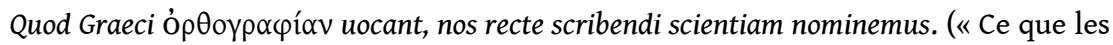
Grecs appellent orthographie, appelons-le "science du bien écrire". »)

Quint. 1.7.1.

12 À ỏ $\rho \theta 0-($ ortho-) correspond l'adverbe recte, à - $\gamma \rho \alpha \varphi$ - (-graph-) les gérondifs scribendi et scientiam servent à faire de l'ensemble un substantif abstrait (orienté vers les noms de science), ce qui était la vocation du suffixe grec -ía (-ia).

13 La différence de traitement tient au fait que l'équivalence univerbée est naturelle si l'on part d'un mot grec doté d'une structure interne radical + suffixe ou préfixe + radical + suffixe (c'est le type auquel appartient prosôdia) mais artificielle quand le mot grec est 
un composé formé de deux radicaux sémantiquement chargés (c'est le type auquel appartient orthographia). On se souvient de la réticence de Cicéron (Top. 35) à proposer une équivalence univerbée au composé etymo-log-ia, pour lequel il forme ueri-loqu-ium « morphème par morphème » (« uerbum ex uerbo », comme il dit ${ }^{5}$ ). Voir à cet égard le commentaire de Quintilien sur le passage cicéronien :

[...] nam uerbum ex uerbo ductum, id est ueriloquium, ipse Cicero, qui finxit, reformidat

(«[...] car le mot tiré morphème par morphème <d'etymologia>, à savoir ueriloquium, même Cicéron, qui l'a pourtant forgé, se refuse à l'employer ».)

Quint. 1.6.28

14 C'est avec ce type de mots composés du grec que les Latins choisissent plutôt de faire correspondre des lexies.

\section{Le calque sémantique}

15 Si le terme grec qu'il faut adapter n'a pas de forme interne analysable, et qu'il est donc rétif au calque morphologique, il reste peut-être la possibilité de faire sur lui un calque sémantique ${ }^{6}$. Ce procédé consiste à utiliser une équivalence sémantique déjà à l'œuvre entre le mot technique grec et un mot latin, qui lui correspond dans un de ses autres sens. Puis, sur ce premier contact, à enrichir le sens du mot latin de celui dont il faut rendre compte dans la langue technique grecque qu'on tente d'adapter. Soit le mot тóroc/topos en grec, qu'on ne saurait décomposer davantage; il signifie «lieu», « endroit » et a comme équivalence de traduction le mot locus. Or Aristote utilise tómoৎ en rhétorique dans un sens nouveau; il n'y a plus qu'à mandater locus en latin pour désigner les lieux de la rhétorique. Les déterminants éventuels n'ont plus qu'à être traduits eux aussi en latin et on crée des lexies techniques sans néologisme: тótrol kolvoí/topoï koïnoï génère la création automatique de loci communes et l'on voit que le français n'a plus qu'à poursuivre le processus avec ses lieux communs.

Autre exemple : les grammairiens grecs nomment les différentes natures de mots $\mu$ ćp

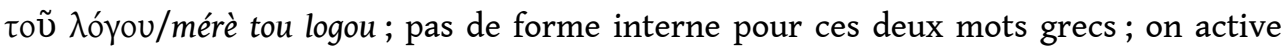
alors l'équivalence sémantique et voici qu'apparaissent les partes orationis, que la Grammaire de Port-Royal (après d'autres) n'a plus qu'à appeler à son niveau les " parties du discours ".

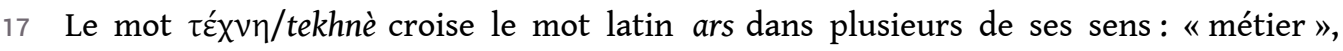
"talent ", "habileté ", "savoir-faire ». Lorsqu'il s'agit de donner un nom à ce genre littéraire, nouveau à Rome, des traités techniques, que précisément les Grecs nomment des tekhnaï (pour nommer la somme des savoir-faire dans un métier donné), les Latins n'ont plus qu'à enrichir ars de ce nouveau sens, par calque sémantique de tekhnè : et voilà que naissent en latin des traités d'art vétérinaire ou d'art culinaire ou des artes poeticae ou des artes grammaticae; et c'est aussi par ce procédé que du latin au français un « homme de l'art » est un technicien.

18 Le calque sémantique est parfois, si ce n'est souvent, le préalable d'un calque morphologique. Ainsi, il est nécessaire d'avoir entériné le sens technique de mores en rhétorique et en philosophie, calqué sur le grec $\tilde{\eta} \theta$ oc/êthos et qui fait passer du sens de " habitudes » à celui de "caractère ", avant de pouvoir proposer de calquer la forme

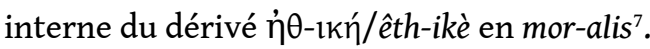


19 Et il se peut aussi que les deux procédés se croisent dans ce que j'appelle un calque morphosémantique. Ainsi quand, pour des raisons sémantiques, on choisit d'exploiter un mot préexistant du lexique qui se trouve avoir en même temps la forme interne du mot dont il se fait le correspondant. Lorsque les grammairiens latins dotent la catégorie $\mathrm{du}$ cas de son nom, il leur suffit de constater que les Grecs, pour cette notion, disent $\pi \tau \tilde{\omega} \sigma ı /$ ptôsis, lequel veut en langue ordinaire dire «chute ». Ils vont donc spécialiser casus qui a le même sens en langue de "chute». Il s'agit d'un calque sémantique, puisque le mot existait déjà et croise un ou des sens antérieurs du terme grec ; mais il a aussi la même forme interne, laquelle signifie «action de tomber». C'est donc un calque morphologique sans néologisme. Participant des deux procédés, il est donc un calque morphosémantique.

Quelque option que choisisse le traducteur du grec en latin, il met du grec dans son latin. Ce grec saute aux yeux et aux oreilles dans le cadre de l'emprunt lexical, avec des traits phoniques parfois incompatibles avec le latin tels que des aspirées, la voyelle $y$, le $\mathrm{z}$, des hiatus à première voyelle longue, des accentuations aberrantes, etc.; le grec est parfois beaucoup plus discret : dans le calque morphologique il se laisse deviner par le choix qui est fait de créer une nouvelle unité lexicale qui n'est latine que d'apparence; dans le calque sémantique il est particulièrement insidieux. Mais il est toujours présent.

\section{Les fluctuations de la métaphore}

21 Il en résulte que, dans un même idiolecte latin, plusieurs solutions terminologiques sont possibles et peuvent coexister. Prenons l'exemple de la métaphore.

Le latin a acclimaté très tôt dans sa rhétorique l'emprunt lexical metaphora; mais il ne s'est pas privé d'en décalquer aussi la forme interne (« de l'autre côté » + "porter » + suffixe de nom d'action), ce qui donne le calque morphologique tra<ns>latio. Et les deux termes, strictement interchangeables, l'un grec, l'autre latin (ou qui donne l'illusion d'être latin), peuvent absolument voisiner dans la même nomenclature, chez le même auteur.

Voici un exemple où Quintilien utilise le terme grec :

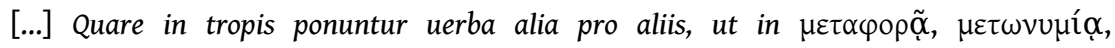

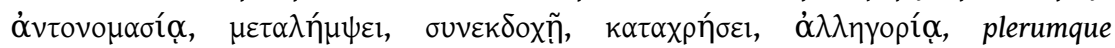

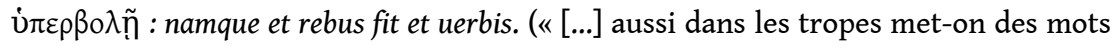
pour d'autres, comme dans la métaphore, la métonymie, l'antonomase, la métalepse, la synecdoque, la catachrèse, l'allégorie, souvent l'hyperbole (car cela se fait autant par les choses que par les mots. »)

Quint. 9.1.5

Un autre (parmi beaucoup) où il utilise tra<ns>latio pour dire la même chose :

Translatio quoque, in qua uel maximus est orationis ornatus, uerba non suis rebus accommodat. («La métaphore non plus, l'un des plus beaux ornements du discours, ne se sert pas des mots appropriés aux choses ».)

Quint. 8.2.6

Et beaucoup d'auteurs techniques profitent de cette double possibilité pour introduire de la variation dans leur énoncé, dans un secteur, la terminologie, où il ne devrait pas y avoir de place pour les variantes. Passe encore qu'on dise incidemment, dans un 
équivalent de notre note de bas de page ${ }^{8}$, le nom de l'autre langue, comme dans ces quelques passages :

[...] metaphoram quam Graeci uocant, nos tralationem. (« [...] ce que les Grecs appellent métaphore et nous translatio. »)

Fest. (Lindsay), p. 136, 23

[...] uae figura dicitur metaphora, id est translatio. («[...] cette figure s'appelle métaphore, c'est-à-dire translatio. »)

Cassiod., Exp. in Ps., 31.185

Quot modis in diuina lege allegoria cognoscitur? M - Quattuor: aut secundum translationem vel metaphoram ut etc. («De combien de façons dans la loi divine reconnaît-on l'allégorie. -Quatre : soit par translation [ou métaphore], par exemple, etc. ».)

Junilius Africanus, Inst. Div. leg.

On peut ici parler d'encyclopédisme, le nom dans l'autre langue pouvant passer pour une information complémentaire voire accessoire; mais au-delà, cela permet notamment de signaler que ce qui s'appelle systématiquement tralatio chez Cicéron s'appelle metaphora en grec mais aussi chez Quintilien et d'autres Latins et qu'il s'agit de deux synonymes interchangeables. Information utile, donc, à un lecteur de ce genre de texte.

Mais la variation est beaucoup plus insidieuse quand, dans le cadre du même exemple, on passe d'un mot à l'autre sans l'appareillage de l'équivalence impliquée par id est, quod dicitur $Y$, etc. Par exemple :

[...] et hoc longe ab illo modo distat qui fit per metaphoram: non enim translatio est cum lex dicitur mens ciuitatis, («[...] et cela est bien différent du type qui se fait par métaphore : car il n'y a pas translation (?) quand on appelle la loi "esprit de la cité" »;)

Marius Victorinus (Stangl), 27.8

[...] beatus uir, qui repleuerit pharetram suam ex ipsis, ut, quia metaphoram semel sumpserat ex sagittis, et in pharetra quoque translatio seruaretur, («[...] "heureux homme, qui aura rempli son carquois de ses propres ressources" (Ps. 127.5), pour pouvoir, puisqu'il avait fait une première métaphore des flèches, filer la métaphore du carquois ».)

Hier. Epist., 34.4

28 À vrai dire, le traducteur devrait s'interdire cette variation et accepter la redite dont l'auteur latin s'est débarrassé sous couleur de changement de langue. Le texte de Marius Victorinus signifie en fait «et cela est bien différent du type qui se fait par métaphore : car il n'y a pas métaphore, etc. ».

Il y a un type d'énoncé intéressant : l'énoncé définitoire, dans lequel l'auteur expose les contours du terme dans son propre système. On sait que la définition lexicographique est toujours circonscrite dans une circularité inévitable: tous les mots qui la constituent sont dans le dictionnaire et les mots qu'on y trouve se définissent les uns les autres, sans échappatoire. Il incombe à l'auteur qui soumet une définition d'en proposer une dont le rayon ne soit pas à courte vue, dans une tautologie immédiate, du type de celle-ci, tirée du Larousse : «Chanteur : Personne qui chante, ou qui fait métier de chanter ».

La définition que donne Aristote de la métaphore n'échappe pas à un certain degré d'évidence. Pourtant Aristote, théoricien de la définition et à qui les lexicographes continuent d'emprunter sa méthode de l'ars definiendi est plutôt adepte de définitions qui ne reprennent pas dans leur énoncé de morphèmes du mot à définir (situation qui

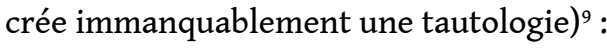




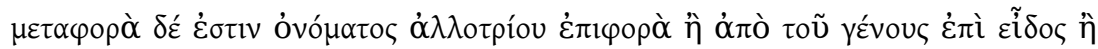

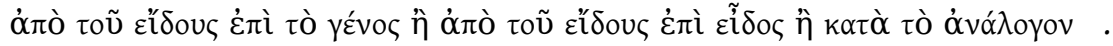

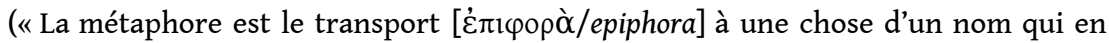
désigne une autre, transport du genre à l'espèce ou de l'espèce au genre, ou de l'espèce à l'espèce ou d'après le rapport d'analogie ».)

Aristt. Po., 1457 b 6

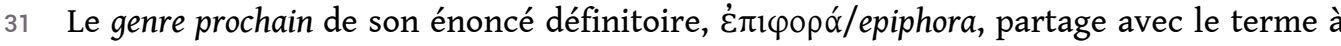

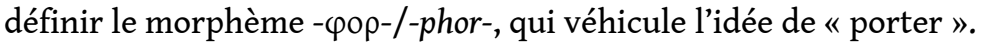

D'autres auteurs techniques, notamment les grammairiens, seront encore moins regardants et n'hésiteront pas à accentuer la tautologie en utilisant dans leur définition

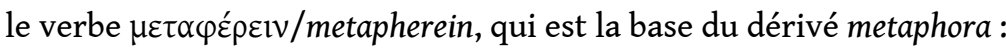

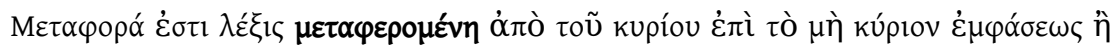

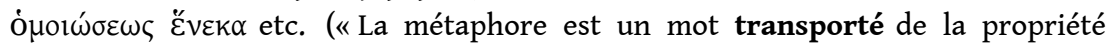
jusqu'à l'impropriété pour faire une emphase ou une comparaison, etc. »)

Tryphon, I, 191.24.

Quand on passe au latin, on s'aperçoit que la traduction de la définition aboutit à un degré plus ou moins visible de tautologie selon qu'on a utilisé comme terme à définir tra<ns>latio ou metaphora. En voici deux exemples opposables :

Translatio est, cum uerbum in quandam rem transferetur ex alia re, quod propter similitudinem recte uidebitur posse transferri ;

Rhet. ad Her., 4.45

Metaphora est uerbi alicuius usurpata translatio etc.

Isid., 1.37.2

Dans la Rhétorique à Herennius, le mot translatio est défini d'une façon telle qu'apparaissent deux occurrences de transfero, verbe dont translatio est le nom d'action dérivé : «Il y a translatio quand se trouvera transféré d'une chose à une autre un mot qui, pour des raisons de ressemblance, semblera pouvoir être correctement transféré ". Par chance, le supplétisme du verbe fero, tuli, latum masque un peu à l'œil et à l'oreille la tautologie morphologique immédiate. Avec l'étymologie d'Isidore, où metaphora est carrément défini par translatio ( « une métaphore est la translation abusive d'un mot »), on a en première instance l'illusion d'avoir affaire à une définition technique. Mais si l'on se souvient de la synonymie complète des deux termes et que l'on pratique la rétroversion du mot le plus latin des deux, on parvient à « la métaphore est la métaphore abusive d'un mot » : la tautologie est au fond, encore plus directe. Elle aboutit à une aporie, semblant induire que la metaphora (terme à définir) est une sorte de translatio (le genre prochain d'Aristote), que metaphora est hyponyme de translatio, translatio hyperonyme de metaphora, avec rupture de l'équivalence synonymique... On ne pourra se tirer d'affaire qu'en admettant qu'ici, il ne faut pas donner à translatio une valeur technique. Ne pas comprendre «la métaphore est une translatio abusive » mais "la métaphore est le transfert abusif d'un mot ». Au fond, chez Isidore, le procédé est comparable à ce qu'on voit dans la Rhétorique à Herennius. L'étymologiste fait une définition tautologique, mais bilingue; mais là où l'auteur de la Rhétorique à Herennius doit limiter sa tautologie à des mots de nature différente (translatio dans le mot à définir et transfero dans l'énoncé définitoire), Isidore peut aller jusqu'à exploiter la tautologie avec des mots de même nature (metaphora et translatio) parce que le bilinguisme crée l'illusion qu'on a bien là deux mots différents! Certes ils le sont, et tous deux figurent dans le dictionnaire latin: mais ils recouvrent une seule et même notion. 
Une dernière errance dans l'utilisation de la terminologie traduite consiste à faire des allusions au terme sans le citer expressément. Ces allusions se comprennent par la forme interne du mot à suppléer, laquelle se trouve présente en filigrane dans l'énoncé. Ainsi, sans citer ni metaphora ni tra<ns>latio, on peut faire référence à la métaphore en utilisant un énoncé qui contient le verbe transfero. Par exemple Donat, dans son commentaire à L'Eunuque de Térence écrit, à propos du vers 771 :

Et haec uerba pro hortatu imperatoris accipienda sunt, quandoquidem omnia de exercitu transferuntur. («Et ces mots doivent s'interpréter comme l'encouragement d'un général, puisque tout est une métaphore militaire $\left.{ }^{10} . »\right)$

Don, ad Eun., 771.2

C'est au traducteur de repérer la variable et, s'il le souhaite, de techniciser sa traduction.

Ces variables où des notions d'ordinaire portées par des substantifs techniques sont véhiculées par des mots non techniques d'autre nature mais qui, en métalangue, disent tout de même quelque chose du technicisme crypté, énigme parfois augmentée d'un changement de langue (comme quand metaphora se cache sous une forme de transfero), apportent aux énoncés latins une forme de dilettantisme qui pourrait agacer l'esprit empreint de logique. Mais les énoncés de ce genre sont fort nombreux et le risque de laisser échapper quelques-unes de ces allusions est grand.

Dans un champ autre que celui de la métaphore, citons deux phrases cicéroniennes qui, heureusement pour nous, sont livrées avec les clés de l'énigme :

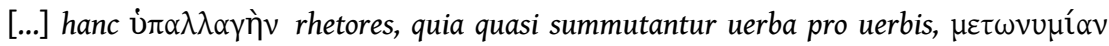
grammatici uocant, quod nomina transferuntur. ([...] «cette figure, les rhéteurs l'appellent hypallage, parce qu'on échange des mots contre d'autres, pour ainsi dire, et les grammairiens l'appellent métonymie, parce qu'on transpose des noms ».)

Cic., Or., 93

[...] iam cum fluxerunt continuae plures tralationes, alia plane fit oratio; itaque genus hoc Graeci appellant $\dot{\alpha} \lambda \lambda \eta$ үopíav. ( «[...] dès lors qu'on fait couler en continu plusieurs métaphores, le discours se fait tout autre; aussi les Grecs appellent ce type allégorie ».)

Cic., Or., 94

Dans Or. 93, deux termes techniques grecs sont cités, présentés par Cicéron comme des variantes idiolectales d'une même notion : l'hypallage des rhéteurs et/ou la métonymie des grammairiens. Notons que Cicéron ne propose pour aucun des deux termes d'équivalent latin. Mais il propose une explication (quia puis quod) de chacune des désignations (uocant). Nous comprenons donc que les deux causales font fonction d'étymologie ou de définition. Pour l'hypallage, l'explication est que quasi summutantur uerba pro uerbis. L'emploi de quasi est notable : Cicéron s'en sert, parfois accompagné d'une forme de quidam, dans les contextes métalinguistiques pour inviter à un retour sur la forme interne d'un mot ${ }^{11}$. Mais le plus frappant reste l'emploi de summutare, verbe très rare chez lui et dans la langue latine. Le rapport de cause à effet instauré par quia n'est pas que «dans les choses »; il est aussi, voire surtout, « dans les mots » et la présence de quasi, délimiteur d'autonymie ou de connotation autonymique, nous incite à comparer les formes internes du terme grec défini et du verbe latin qui l'explique. Or ils ont une forme interne identique au moins pour la partie sémantiquement chargée : «dessous» ( $\dot{0} \pi<0>-/$ hyp<0>-, sub-) + «échanger» (- $\alpha \lambda \lambda \alpha \gamma_{-}-/$-allag-, - mut-). L'explication de la métonymie, quant à elle, exploite aussi la forme interne du terme grec, cette fois dans l'ordre inverse des morphèmes à traiter : quia nomina (- $\omega v v \mu-/-$ 
ônym-) transferuntur ( $\mu \varepsilon \tau-/ m e t-)$. Le choix des mots latins qui définissent les termes grecs en décode la forme interne.

Dans Or. 94, c'est le lien logique de cause à effet instauré par itaque qui nous oblige à interpréter le rapport annoncé entre alia plane fit oratio, "le discours se fait tout autre », et le terme $\dot{\alpha} \lambda \lambda \eta \gamma$ үí́ $\alpha$ /allêgoria. Et nous le comprenons seulement en activant

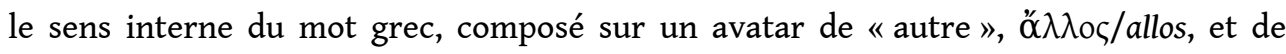

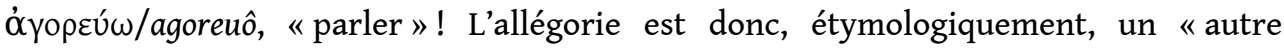
discours ». Sans la mention explicite du nom du trope, l'allusion alia oratio risquerait de rester longtemps énigmatique. Mais là où, sans que figure explicitement le terme allegoria, on trouve la lexie alia oratio, ne faudrait-il pas voir une manière non technique (dilettante, agaçante, etc.) d'y faire néanmoins allusion? Ainsi dans Tite-Live, Camille, à la fin du Livre $\mathrm{V}$, se livre à un long discours pour persuader les Romains de réintégrer Rome et de la reconstruire, contre l'avis des tribuns. À la fin de son discours, le récit reprend sur ces mots :

Mouisse Camillus cum alia oratione tum ea, quae ad religiones pertinebat, maxime dicitur.

("Camille, dit-on, obtint une vive émotion d'une part avec son autre discours (?),

d'autre part avec celui qui évoquait la religion ».)

Liv. 5.55.1

Or il n'y a pas de premier discours... S'agit-il simplement de l'emploi pragmatique d'alius par lequel on présente dans un ordre croissant de force des arguments : " par ce discours, et particulièrement la partie sur la religion »? Ou s'agit-il de dire (sans le dire) que c'était une allégorie ("Camille, dit-on, obtint une vive émotion par son allégorie et surtout le passage sur la religion ») ? La même question peut se poser au Livre XLV (Liv., 45.44.19) : le roi Prusias vaincu se montre à son désavantage, en bonnet d'affranchi, la tête rasée, et tient au Sénat un discours aussi flatteur pour ses auditeurs que honteux pour lui («aliamque orationem non tam honorificem audientibus quam sibi deformem habuisse»). Pourquoi un "autre discours»? Pourquoi ne pas comprendre plutôt qu'« il fit une allégorie moins flatteuse pour son auditoire que déshonorante pour lui-même »?

\section{La migration de la terminologie gréco-latine dans les langues vernaculaires}

Après cette incursion panoramique, nécessairement biaisée par son extrême généralité, voyons, avec le même défaut de ne s'intéresser qu'à des tendances, comment cette terminologie bilingue s'acclimate dans les langues modernes, en tout cas en français.

Le français est dans la situation du latin par rapport au grec: au moment de faire dans sa langue un traité des tropes, par exemple, l'auteur dispose de la longue histoire latine de la discipline et de son appareil terminologique. Mais cette fois, le point de départ est une terminologie double, une latine, l'autre grecque. Les plus savants, par la connaissance qu'ils ont aussi du grec, disposent même des sources de leurs sources latines. La théorie et la terminologie en français vont donc être influencées par les deux langues antiques. Mais les grammairiens français vont parfois avoir à cœur de traduire en français (par calque morphologique ou sémantique) le double mot gréco-latin de l'Antiquité, créant ainsi un troisième terme pour rendre compte a priori de la même notion. 
L'innovation moderne est que, face à des termes techniques sanctifiés par le temps et, le cas échant, augmentés d'un parasynonyme français, les techniciens de l'art oratoire vont être tentés de tout garder et d'ordonner la profusion. J'en prendrai deux exemples grammaticaux.

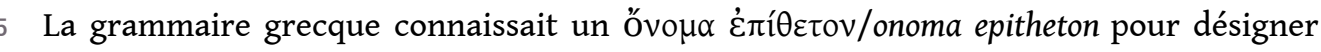
une sorte de nom; pour cette lexie, les Latins ont systématisé le calque sémantique nomen (qui croise onoma au sens de "nom d'une personne ») auquel ils ont adjoint soit l'emprunt lexical epithetum, soit un calque morphologique qui rende

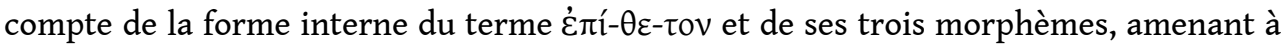

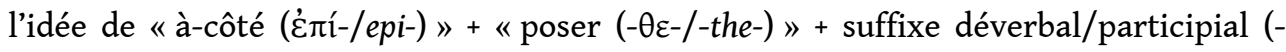

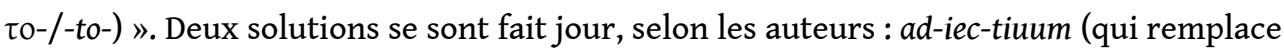
l'idée de "poser» par le plus brutal "jeter ») et ad-posi-tum. Et de ces trois variantes synonymiques nomen epithetum / adiectiuum / appositum, le français (lorsque, au Moyen Âge, l'adjectif a été inventé comme nature de mot distincte du substantif) a tiré épithète, adjectif et apposé, et les a spécialisés dans un système rénové et amélioré, l'un devenant nom de nature, les deux autres noms de fonction.

De même, parmi les pronoms, existait une espèce nommée en grec óvapopıkóv/ anaphorikon. Les Latins vont donc connaître un pronomen anaphoricum par emprunt lexical mais aussi un pronomen relatiuum, par calque morphologique traduisant, morceau par morceau et dans le même ordre, les composants du terme grec (ana$=r e^{-12}$, «de nouveau "; -phor- $=-$ lat $^{-13}$, «porter $" ;-i k o n=-$ tiuum $\left.^{14}\right)$. Ajoutons à ce doublon les variantes francisées de ré-fér-ent ou de rapport (tous mots qui ont le même sens parallèle, celui qui surgit en métalangue), et l'on constate que c'est à la grammaire française de voir désormais comment améliorer son système pour faire place aussi bien à un anaphorique qu'à un relatif, à un référent, à un rapport. C'est ce que j'appelais plus haut les « accidents bénéfiques ».

Pour revenir à la nomenclature rhétorique et pour disposer facilement d'un corpus de termes avec leurs définitions, je choisis de limiter mon étude à trois grands recueils français : celui des Tropes de Dumarsais, de 1730, lu dans l'édition commentée que Fontanier en a donnée en 1818 (et que je simplifierai désormais en DM, 1818) ; ceux de Fontanier, en deux volumes de 1821 et 1827, réunis dans Les Figures du discours chez Garnier-Flammarion en 1977 (d'où Fontanier, 1977) et celui de Dupriez, Gradus, dictionnaire illustré par l'exemple des procédés littéraires ${ }^{15}$.

Le français a donc dans son stock de termes des mots d'origine grecque dont il s'astreint à préserver la grécité dans son système de translitération orthographique (hérité des Latins) : des rh- initiaux, des digrammes ph, th, ch, le $y$, le $z$, etc., là où d'autres langues romanes ont plutôt modernisé l'orthographe de ces hellénismes et donc amenuisé leur caractère étrange. Le mot français correspondant a d'emblée une allure plus pédante que ses correspondants italiens ou espagnols : synalèphe, chleuasme, $z_{\text {zeugme }}{ }^{16} . .$. Il possède aussi des mots latins, mais de la filière savante de ceux qui ne sont pas passés par le filtre de la transmission orale continue et qui sont, ni plus ni moins, des emprunts au latin à peine retouchés dans leur orthographe: annomination, prétérition, interjection, etc. Et il a donc aussi des doublons, j'entends par là des couples formés d'un mot grec et d'un mot latin censément synonymes, le terme latin étant le calque (morphologique ou sémantique) du terme grec. C'est à quelques-uns de ces doublons que je voudrais consacrer ces dernières lignes, pour apprécier comment le 
système moderne s'accommode de ce scandale théorique qu'est la synonymie dans le cadre d'une nomenclature technique.

En règle générale, dans un système donné, il n'y a pas de place pour un doublon synonyme. Lorsque le terme latin et le terme grec coexistent chez un même auteur, ils sont dans une relation hiérarchisée (l'un est l'hyperonyme de l'autre), complémentaire ou nulle.

\section{Translation et métaphore (et transfert ?)} ressemblance », objet du chapitre III de la première section («Des Tropes en un seul mot, ou proprement dits ») de la seconde partie («Théorie des Tropes ») du Manuel pour l'étude des tropes de 1821. Le grammairien y précise (Fontanier, 1977, 99 sq.) que : « Les Tropes par ressemblance [...] se réduisent, pour le genre, à un seul, à la Métaphore, dont le nom si connu, et plus connu peut-être que la chose même, a perdu, comme l'observe Laharpe, toute sa gravité scolastique ", raison pour laquelle, sans doute, il se dispense d'en donner une définition technique. Mais dans une paraphrase définitoire qu'il donne en passant lors de cette section, on retrouve l'allusion au sens étymologique :

La Métaphore d'une chose animée à une chose inanimée ; c'est-à-dire le transport à une chose inanimée de ce qui est le propre d'une autre chose inanimée. (Fontanier, 1977, 101)

chez Dupriez (1984), les deux notions sont toutes deux des entrées spécifiques mais ne se recouvrent pas du tout sémantiquement. La métaphore correspond à l'idée qu'on s'en fait. Mais la translation (s.v.) est un « changement de catégorie grammaticale avec ou sans marquant» (variantes synonymiques annoncées chez d'autres auteurs: dérivation impropre, hypostase, transfert). Et Dupriez précise que: "Bary ${ }^{17}$ donne translation comme synonyme de métaphore (t. 1, p. 274) ». Signalons pour mention que le terme transport (qui véhicule lui aussi le même sens interne que métaphore, translation et transfert) est, chez Dupriez, mentionné comme un équivalent d'« exaltation délirante » dans l'article « réactualisation » (p. 379).

Les deux termes métaphore et translation, donc, dans notre corpus technique français, soit ne cohabitent pas (Dumarsais), soit cohabitent discrètement (Fontanier, expliquant le sens étymologique de métaphore) soit cohabitent mais restent sans relation (Dupriez). 


\section{Périphrase et circonlocution} (Quint. 9.3.66) : hinc est $\pi \alpha \rho o v o \mu \alpha \sigma i ́ \alpha$, quae dicitur adnominatio (« de là vient la paronomase, qui se dit en latin adnominatio »). Il n'a rien pour surprendre car la

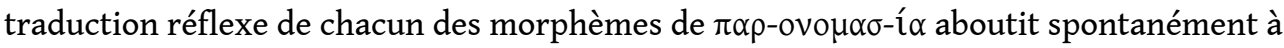
ad-nomina-tio.

Pas de doublon en revanche chez nos grammairiens de l'âge classique. Dumarsais et Fontanier ne pratiquent que la paronomase. Dupriez, en revanche, accorde une entrée spécifique à chaque notion. Mais ils ne sont pas mis en rapport, hormis dans une remarque de l'article « annomination » (p. 48) où il est dit que «Scaliger, Marouzeau et Lausberg [...] font d'annomination un synonyme de paronomase» et en retour, sous "paronomase ", où annomination est indiqué comme synonyme chez les mêmes auteurs. 
L'analogie existe implicitement : la paronomase concerne des noms, l'annomination des noms propres. Cette distinction moderne, sans modèle antique ${ }^{19}$, transformant sans doute (implicitement) l'annomination en catégorie de la paronomase, donc en hiérarchisant les deux notions, rend compte de ce que nous voulons montrer : on garde les deux éléments du binôme mais en les spécialisant différentiellement.

\section{Pronomination et antonomase}

Les Latins ont utilisé le terme d'antonomasia pour désigner l'antonomase de nom propre et n'ont pas songé à décalquer le terme en latin. Ils en ont plusieurs fois illustré la forme interne ${ }^{20}$ mais n'ont pas été (pourquoi ?) jusqu'à créer le pro-nomina-tio qui serait le fidèle sosie latin de ant-ônomas-ia $a^{21}$ («à la place de » + "nom » + suffixe de nom d'action). Ce n'est qu'au Moyen Âge que Sedulius Scottus, dans son commentaire à la grammaire de Donat, propose l'équivalence bilingue: Antonomasia est significatio uice nominis posita. quae pronominatio interpretari potest («L'antonomase est un mot mis à la place d'un nom; on peut le traduire pronominatio ", Sedulius Scottus, In Donati artem maiorem, 3.381.13 [Löfstedt]).

Dumarsais $(1818,132)$ n'a adopté que l'antonomase, dont il donne une définition discrètement étymologique: "L'antonomase est une espèce de synecdoque, par laquelle on met un nom commun pour un nom propre, ou bien un nom propre pour un nom commun ».

Fontanier $(1977,95)$ a une définition qui n'est pas tautologique (si l'on en excepte la présence du mot nom, évidemment) mais peu après il en donne des espèces qui présentent la même formulation étymologique que Dumarsais : « $\mathrm{I}^{\circ}$ Un nom commun pour un nom propre », etc. Et il connaît aussi la pronomination, qui est chez lui tout autre chose que l'antonomase et qui s'apparente à la circonlocution ${ }^{22}$ et qu'à ce titre, il faut distinguer de la périphrase.

On trouve aussi le doublon chez Dupriez (1984), mais très indirectement. En effet, " antonomase " est une entrée ; quant à pronomination, il figure sous "périphrase ", avec un renvoi à Fontanier (cité ci-dessus) qui invite à distinguer la périphrase de la pronomination.

\section{Prosopopée et personnification}

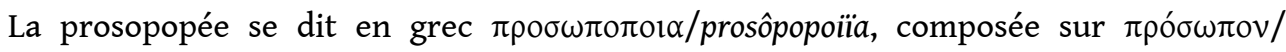
prosôpon (" masque »), le radical du verbe $\pi$ or $\omega^{\omega} /$ poieô ( faire ») et un suffixe de nom d'action. Un calque sémantique préalable de persona sur $\pi \rho o ́ \sigma \omega \pi$ ov («masque », d'où " personnage » et « personne ») permet la création en latin du composé personi-fica-tio, qui décalque scrupuleusement les morphèmes du grec. Sauf que ce n'est pas un mot qui a été créé en latin... Il existe en néolatin, chez Raymond Lulle, un adjectif personificabilis et le verbe personificare, décalqués du grec; mais les Latins authentiques ne l'ont pas inventé. Eux se contentent de l'emprunt prosopopoea et en expliquent le sens interne dans des équivalences ou des définitions ${ }^{23}$. Le mot personnification est donc inventé en français (ce que trahit d'ailleurs sa géminée) mais comme s'il était latin! Reste qu'il est le doublon de prosopopée et qu'il faut donner une place à chacun.

Dumarsais connaît la prosopopée mais non la personnification, même si le verbe personnifier figure dans son traité. 
insensible, ou d'un être abstrait et purement idéal, une espèce d'être réel et physique, doué de sentiment et de vie, enfin ce qu'on appelle une personne». On notera, par le choix typographique de l'alternance des italiques et des romains (que je respecte dans ma citation et qui est de Fontanier) la volonté de faire parler le sens interne du terme. En résumé, et en focalisant sur les caractères en romain, hormis ceux du terme à définir, on a : «La personnification consiste à faire <une> personne», soit une définition bien tautologique, très comparable aux énoncés techniques antiques que nous avons croisés plus haut. La personnification de Fontanier est un «trope en plusieurs mots ou improprement dit». La prosopopée (Fontanier, 1979, 404), elle, est une «figure de discours autre que les tropes » et Fontanier la définit ainsi : «La Prosopopée, qu'il ne faut confondre ni avec la Personnification, ni avec l'Apostrophe, ni avec le Dialogisme, qui l'accompagnent presque toujours, consiste à mettre en quelque sorte en scène les absents, les morts, les êtres surnaturels, ou même les êtres inanimés, etc. ». On voit que le premier réflexe de Fontanier est de faire immédiatement un distinguo avec la personnification, et cela en raison de l'identification inévitable qui peut en être faite par qui regarde la formation des deux mots. La personnification accompagne la prosopopée, elle semble en être un outil mais non une subdivision; le rapport entre les deux termes est donc un rapport d'analogie ou de corrélation.

Chez Dupriez (1984), chaque terme a son entrée propre et il n'y a pas de renvoi de l'un à l'autre : la relation y est donc nulle.

Il est temps de conclure. Le panorama rapide présenté ici est bien imparfait. Nombre de termes échappent à la traduction littérale (synecdoque $e^{24}$ ) ou trouvent un correspondant sans rapport morphologique : épitrope = permission (Fontanier). Mais la tendance est tout de même assez visible : on a à cœur d'exhiber le sens interne du terme à expliquer, on est tenté de lui trouver un équivalent dans l'autre langue et, quand on a décidé de garder les deux mots du doublon dans son système, on tâche d'affiner ledit système pour éviter une synonymie pure, qui relève de la faute technique. Ainsi, en partant des termes de Fontanier, voici, pour mention (et sans prétendre à l'exhaustivité), d'autres doublons qu'on peut répertorier chez au moins l'un de nos trois auteurs modernes (et qui n'offrent parfois aucune relation entre les deux exemplaires du doublon):

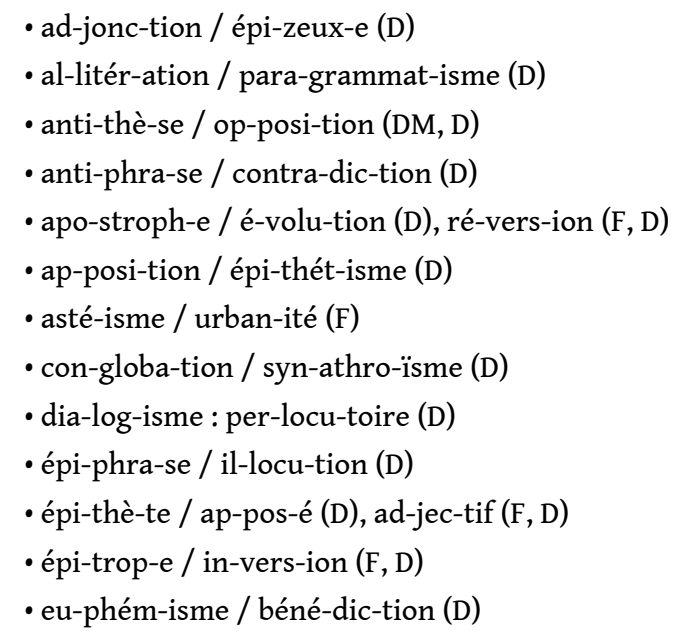

Recherches \& Travaux, 99| 2021 
- imita-tion / mimè-se (D)

- méta-bol-e / tra-jec-tion (D)

- méta-lep-se / tran-sump-tion (D)

- para-phra-se / al-locu-tion (D)

- pré-occupa-tion / pro-lep-se (F, D)

- ré-tic-ence / apo-siopè-se (D)

- syl-lepse / con-cept (D), con-cep-tion (DM, D), com-préhen-sion (DM, D)

- syn-thè-se / com-posi-tion (D)

- trope / tour (F, DM, D)

- zeug-me / jonc-tion (D)

Cette situation n'est pas réservée à la langue de la rhétorique. Elle a cours dans le vocabulaire de la critique littéraire ${ }^{25}$. Je fais l'hypothèse que toutes les sciences qui remontent à l'Antiquité sont dans cette situation d'avoir une double terminologie gréco-latine parfois augmentée d'une vernaculaire: la médecine connaît des prodromes, signes pré-curseurs ou symptômes avant-coureurs d'une maladie; quel est le rapport lexicologique de péri-ple à circum-navigation; de péri-phér-ie à circon-fér-ence; de hypo-tax-e à sub-ordina-tion; de syn-tax-e à co-ordina-tion ; de héliotrope à tourne-sol, etc. ? Gageons que chaque idiolecte, soucieux de garder ces paires de jolis synonymes issus des deux langues savantes de l'occident, leur fait artificiellement une place dans sa nomenclature, en distinguant techniquement les deux éléments du diptyque.

\section{NOTES}

1. Voir par exemple Quint., Inst., 3.1.8 : "Artium autem scriptores antiquissimi Corax et Tisias Siculi, quos insecutus est uir eiusdem insulae Gorgias Leontinus, Empedoclis, ut traditur, discipulus » («Les plus anciens auteurs de traités, Corax et Tisias de Sicile, que poursuivit un homme de la même île, Gorgias de Leontium, un disciple d'Empédocle, à ce qu'on dit »). Je renvoie pour un aperçu de la naissance de l'art de la rhétorique aux introductions des éditeurs critiques de la Rhétorique d'Aristote, particulièrement M. Dufour pour la C.U.F et J. H. Freese pour la Loeb Classical Library.

2. Aristt., Rhet, 1354 a-12.

3. M. Dufour, Aristote, Rhétorique, Paris, C.U.F., Les Belles Lettres, 1967, Introduction générale, p. 6.

4. Voir J. Rey-Debove, «La sémiotique de l'emprunt lexical», Travaux de Linguistique et de Littérature, 11-1 (= Mélanges Paul Imbs), 1973, p. 109-123 et C. Nicolas, Sic enim appello... Essai sur l'autonymie terminologique bilingue chez Cicéron, Louvain-Paris-Dudley, MA., Peeters, 2005, p. XIIXXVIII.

5. Sur cette expression uerbum ex uerbo et le concept de verbumex verbalité, cf. C. Nicolas, Sic enim appello..., ouvr. cit., p. 96-138.

6. Sur ce procédé, cf. C. Nicolas, Utraque Lingua. Le Calque sémantique, domaine gréco-latin, Louvain, Peeters, 1996.

7. Cicéron explique précisément tout ce mécanisme psycholinguistique dans les tout premiers

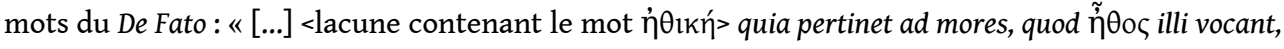
nos eam partem philosophiae de moribus appellare solemus, sed decet augentem linguam Latinam 
nominare moralem ». («[...] puisqu'elle participe des mœurs, que les Grecs appellent $\tilde{\eta} \theta$ o, nous, cette partie de la philosophie, nous l'appelons philosophie des mœurs mais il convient que nous augmentions le vocabulaire pour l'appeler moralis ».

8. Voir, sur ce procédé qui consiste à donner incidemment un synonyme bilingue, C. Nicolas, « La Note de traducteur dans l'Antiquité et le niveau méta- de la traduction, ou Quand la patte du traducteur se prend dans le fil du texte ", dans B. Bortolussi, M. Keller, S. Minon et L. Sznajder, Traduire, transposer, transmettre dans l'Antiquité gréco-romaine, "Textes, Images et Monuments de l'Antiquité au haut Moyen Âge ", nº 9, Paris, Picard, 2009, p. 61-89.

9. Voir par exemple, dans la Rhétorique, ses définitions techniques des quatre types de

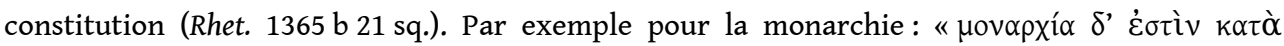

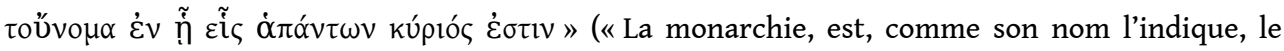
régime où un seul est le souverain de tous »), on le voit résister, bien qu'il soit dans l'évidence du

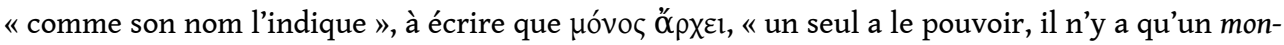

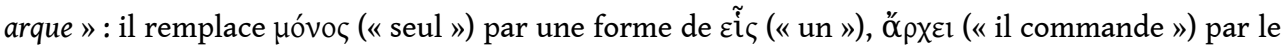
tour kúpıó $\dot{\varepsilon} \sigma \tau \imath v$ (« il est maître »).

10. Traduction empruntée à notre site <http://hyperdonat.huma-num.fr/>.

11. Voir C. Nicolas, Sic enim appello..., ouvr. cit., p. 49-54.

12. Équivalence réflexe, qui aboutit à de nombreux doublons bilingues comme ana-mnè-se / remémora-tion, ana-diplo-se / ré-duplica-tion, etc.

13. Selon le même rapport qui unit metaphora à translatio : cf. supra.

14. Comme par exemple dans tous les noms latins des cas nomina-tiuus, etc., tous (jusqu'au datif) bâtis comme leur modèle respectif en grec, toujours (au féminin) en -ıkń.

15. Donc respectivement C.Ch. Dumarsais, Des Tropes ou des Diférens sens dans lesquels on peut prendre un même mot dans une même langue, Paris, Veuve Brocas, 1730, lu dans l'édition de Fontanier [1818]: P. Fontanier, Les Tropes de Dumarsais, avec un commentaire raisonné [...] par M. Fontanier [...], Belin-Le Prieur, 1818, disponible sur Gallica (<https://gallica.bnf.fr/ark:/12148/ bpt6k50577z/f67.item>) ; P. Fontanier, Les Figures du discours, Paris, Garnier-Flammarion [rééd.], 1977 ; B. Dupriez, Gradus. Les Procédés littéraires (dictionnaire), Paris, 10/18, 1984.

16. Termes qui participent du «jargon ou fatras scolastique » évoqué par Fontanier $(1977,259)$.

17. Auteur d'un traité théorique au XVII ${ }^{\mathrm{e}}$ siècle.

18. Fontanier $(1977: 361)$.

19. Ainsi Quintilien 9.3.70, illustrant la figure de $\pi \alpha \rho o v o \mu \alpha \sigma i ́ \alpha / a n n o m i n a t i o$, donne des exemples d'adjectifs, de substantifs, de verbes, mais aussi d'un nom propre : « "amari iocundum est, si curetur ne quid insit amari." "auium dulcedo ad auium ducit", et apud Ouidium ludentem: "cur ego non dicam, Furia, te furiam?” " ("être aimé [amari] est agréable s'il ne s'agit pas de mettre là de l'amer [amari]; la douceur des oiseaux [auium] mène à l'inaccessible [auium]; et chez le ludique Ovide : "Pourquoi, Furia, ne te dirais-je pas furie ?" ».)

20. Par exemple Quint. 8.6.29: «Antonomasia, quae aliquid pro nomine ponit » ("L'antonomase qui met quelque chose à la place d'un nom »); Isid. 1.37.11, "Antonomasia est pro nomine, id est uice nominis posita " («L'antonomase se met en guise de nom, c'est-à-dire à la place d'un nom »); etc.

21. Hormis l'auteur de la Rhétorique à Herennius 4.31.42 qui, lui, n'utilise pas antonomasia.

22. Fontanier $(1977,326)$ : «Si on ne veut pas de ce nom de Pronomination, qui cependant me parait assez convenable, je ne vois que celui de Circonlocution pour le remplacer ».

23. Quint. 6.1.25: «[...] prosopopoeiae, id est fictae alienarum personarum orationes » («[...] prosopopée, à savoir des discours fictifs de personnages étrangers») ; Isid. 2.21.45 : «Prosopopoeia est, cum inanimalium et persona et sermo fingitur ». (« Il y a prosopopée quand, pour des inanimés, on forge un personnage et son discours. ») Ce qui rend le latin rétif à la création de personificatio, c'est que le mot de départ est un composé en grec, situation où le latin hésite à 
décalquer la forme interne (comme dans le redoutable ueriloquium pour etymologia, discuté plus haut).

24. Fontanier $(1977,261)$ s'y essaie mais le com-préhen-sion ou le con-cep-tion qu'il propose ne tient pas compte du sens « recevoir » qu'on a dans le second radical grec, ni du préfixe -ec-.

25. Voir récemment sur une question de terminologie de la dramaturgie C. Ailloud-Nicolas et C. Nicolas, « Comment la traduction d'un technicisme peut faire évoluer le concept : l'exemple de la péripétie aristotélicienne et de ses avatars en latin et en vernaculaire ", dans M. Taillade, J. Gallego, F. Fatello, G. Gibert (dir.), Nemo par eloquentia: Mélanges de linguistique ancienne en hommage à Colette Bodelot, Presses Universitaires Blaise-Pascal, Cahiers du Laboratoire de Recherche sur le Langage, 2019, p. 521-540.

\section{RÉSUMÉS}

La communication interroge le passage de la nomenclature technique de la rhétorique du grec au latin, puis du latin au français. Les procédés utilisés sont classiques: emprunt lexical, calque morphologique, calque sémantique. Mais comme ils ne sont pas exclusifs, on obtient souvent en latin des doubles listes, une grecque, acquise par emprunt lexical (ainsi metaphora), l'autre latine, issue d'un calque (ainsi translatio), avec deux mots pour la même notion. Et à date française, ce double héritage gréco-latin laisse de nombreuses traces dans la terminologie.

This paper deals with how the list of technical words or Rhetorics goes from Greek to Latin, first, then from Latin to French. The psycholinguistic processes involved are well known: lexical borrowing, calque, semantic borrowing. But, as they are not exclusive, is attested often in Latin a double list, one which sounds Greek, issued of lexical borrowing (e.g. metaphora), the other which sounds Latin, issued of calque (e.g.translatio): two words for one notion. In French, this dual Greek-Latin heritage leaves many traces in the terminology.

\section{INDEX}

Mots-clés : grec, latin, terminologie, métaphore, transfert linguistique, traduction, nomenclature, bilinguisme.

Keywords : Greek, Latin, terminology, metaphor, linguistic transfer, translation, list of terms, bilingualism

\section{AUTEUR}

\section{CHRISTIAN NICOLAS}

HiSoMA, Université Jean Moulin Lyon 3 - UDL

Christian Nicolas est professeur de langue et littérature latines à l'Université Jean Moulin-Lyon 3 (Université de Lyon) et membre de l'UMR HiSoMA. Spécialiste de la littérature de commentaire et co-responsable scientifique du programme Hyperdonat (voir <http://hyperdonat.huma-num.fr/ $>$ ), il a écrit sur le bilinguisme gréco-latin dans l'Antiquité de nombreux articles et deux 
monographies : Utraque lingua. Le calque sémantique : domaine gréco-latin, Bibliothèque d'Études Classiques, $\mathrm{n}^{\circ}$ 10, Peeters, Louvain/Paris, 1996 et Sic enim appello... Essai sur l'autonymie terminologique gréco-latine chez Cicéron, BEC nº 45, Peeters, Louvain/Paris, Dudley Ma., 2005. 